\title{
Subject Index Vol. 140, 2006
}

\section{Allergy Immunology}

Acute poststreptococcal glomerulonephritis 9

Adenoid 223

Adhesion molecules 113

Airway inflammation 215

Albumin 113

Allergen 73, 131

- extracts 30

Allergic asthma 245

- rhinitis 223

- rhinoconjunctivitis 121

Allergy 157, 321

- vaccine 295

Anaphylaxis 27

Antiallergic food 306

Antibody production 306

Antigen presentation 53

Antihistamine 315

Antimicrobial peptides 103

Apoptosis 277

Asthma 96, 139, 321

-, murine model 215

Atopic eczema 239

Atopy 89

- patch test 239

Autoimmunity 174

Basophils 150, 252

Bifidobacterium 157

Blocking antibodies 261

Bronchial responsiveness 327

Buckwheat 73

BWp16 73

Carbohydrate antigens 96

CCL11 205

CCL24 205

CD18 139

CD4+CD25+ T reg 252

CD40 ligand 1,150

- - gene 1

- /CD40L 53

CD40L 342

CD49b 139

Challenge test, oral food 170

Chemokine receptor 345

Chemokines 205

Chemotaxis 103, 113, 315

Chronic nasal polyposis 205

Common variable immunodeficiency 199, 342

Contact dermatitis 231
Supplement issue No. 1 has its own Subject Index
Corticosteroid 199

Cough reflex sensitivity 327

Coumarin 231

Cross-reactivity 30

Cross-sensitization 170

CTLA4 expression 223

Cytokines, type 2270

Cytotoxic T lymphocytes 53

DBPCFC 252

Dendritic cell 53

Dermatophagoides farinae 295

- pteronyssinus 295

Desensitization 20

Desloratadine 315

Drug allergy 164

- hypersensitivity 20

- provocation test 164

Dystonia 27

Eczema 157

Enterococcus 157

Eosinophil 113, 334

- protein X 334

Eosinophilia 205

Eosinophilic inflammation 327

Eosinophils 103, 277

Eotaxin 205

Epinastine hydrochloride 43

Epinephrine 27

Epitopes 285

EPO 277

Escitalopram 27

FasL expression 223

Fc receptor 53

Fecal samples 334

Finland 89

Flavonoids 150

Food allergy 36, 170, 270

Food-induced gastrointestinal hypersensitivity 334

Fungi 30

1,3- $\beta$-Glucanase 131

Glycoproteins 30

Grass pollen allergy 121

Gut microbiota 157

H1 receptor antagonist 43

- $\quad$ gene knockout mouse 215

Helicobacter pylori 89
Hepatitis A 89

Histamine 215

House dust mite 295

Human peripheral blood CD4+ T cells 43

Hygiene hypothesis 89,157

ICOS 342

IgA 96

IgE 270

-, specific 245

$\mathrm{IgG}_{2} 96$

Immediate hypersensitivity 20

Immunoblot inhibition 170

Immunodeficiency 199

Immunoglobulin 185

- E 36, 121

Immunoglobulin-E-binding region 285

Immunotherapy 131, 245, 321

Infection 199

Infections 89

Inflammatory cells 345

- diseases 174

Innate immunity 103

Interleukin 10139

Interleukin-12 277, 306

Intestine 199

Intravenous immunoglobulin 185

- $\quad$ therapy 1

Karelia 89

Keratinocytes 315

Leukotriene $\mathrm{E}_{4} 334$

- stimulation test 20

Local lymph node assay 231

Lolium multiflorum 121

- perenne 121

Loratadine 315

Lower airway remodeling 327

Lung 261

- inflammation 103

Lymphocyte transformation test 20

Lymphoproliferative response 82

Mannan-binding lectin 9

Mast cell degranulation 164

Matrigel 277

Maturation 53

Migration 53, 113, 277

Mould 30

Mucosal protection 96

Multiple sclerosis 174 
Naïve T cells 82

Nasal allergy 327

Neutrophils 103

N-methyl histamine 252

Occupational wheat allergy 252

Ole e 9 quantitation 131

Olea europaea 131

Olive pollen allergy 131

Opium 170

Oral tolerance 306

pDC 252

Perfume allergy 231

Phl p 5b 285

Pimecrolimus 239

Pneumocystis jiroveci pneumonia 82

Pollen allergen 285

Prick-puncture skin test 245

Protein $\mathrm{H} 9$

RACE 73

Recombinant protein 73
Regulatory T cells 174

Rhinitis 321

Rifampicin 20

Russia 89

Scleroderma 345

Sesame 270

Signal peptide 73

- transduction 113

Sinusitis 205

Site-directed mutagenesis 285

Skin tests 20

Standards 185

Streptococcal cysteine proteinase 9

- inhibitor of complement 9

Strongyloidiasis 199

Sublingual immunotherapy 321

Survival 277

T helper type 2 response 215

- regulatory type 1 cells 139

Th1/Th2 cell 53

Th2-type cytokine 43
Therapeutic use 185

Tolerability 321

Toxicity 185

Transcription factor 174

Tryptase 164

Tumor 53

Updosing 321

Urinary samples 334

Urticaria 20

Vaccination 261

Wheat protein hydrolysate 36

$\mathrm{X}$-linked agammaglobulinaemia 82

- - M syndrome 1

Yeast 30 